\title{
Medium dose rate endobronchial radiotherapy with caesium-137
}

\author{
P J M George, J M Hadley, B S Mantell, R M Rudd
}

Endobronchial radiotherapy has considerable potential as a palliative treatment in patients with tracheobronchial malignancy. Placing of a suitable radioactive source adjacent to an endobronchial tumour enables high doses of radiation to be delivered locally with negligible doses to surrounding normal tissues. ${ }^{1-5}$ The aim of treatment is to obtain long lasting relief from endobronchial obstruction while avoiding appreciable radiation damage to lung parenchyma.

The most sophisticated techniques use high activity radioactive sources that are loaded by remote control into prepositioned catheters within the airway. ${ }^{3-5}$ Facilities for delivering this form of treatment, however, are available in only a few centres in the United Kingdom. We have developed a method for delivering medium dose rate endobronchial radiotherapy using caesium-137 $\left({ }^{137} \mathrm{Cs}\right)$. This technique uses standard equipment used to treat gynaecological malignancy and has the advantage of being readily available. The method and our preliminary results are reported in this paper.

\section{Management of patients with large airway obstruction}

Patients with inoperable bronchial tumours were initially assessed for endoscopic laser treatment. If their main symptoms were thought to be caused by intraluminal tumour they were treated with the laser, and if they derived obvious clinical benefit from this they proceeded to endobronchial radiotherapy after an interval of one to two weeks, the aim being to prolong the period of airway patency obtained with laser therapy.

All patients were treated with a neodymium YAG laser operating at a wavelength of $1.32 \mu \mathrm{m}$ (MBB, Medilas 2, Medizintechnic). Treatments were given under intravenous general anaesthesia with a combination of rigid and flexible bronchoscopes. Full details of this method have been published elsewhere. ${ }^{6}$

\section{Endobronchial radiotherapy}

INSERTION OF AFTERLOADING CATHETERS

Patients were readmitted within two weeks of laser treatment and underwent rigid broncho- scopy under general anaesthesia. Residual tumour and fibrin were debrided from the airway with biopsy forceps. The fibreoptic bronchoscope was then passed inside the rigid bronchoscope and the endobronchial extent of the tumour was measured. The rigid bronchoscope was replaced with a ventilating tracheoscope (Storz) and ventilation was maintained with a jet ventilator. The tip of the tracheoscope was held immediately below the vocal cords while a minitracheotomy incision was made through the cricothyroid membrane. An intravenous cannula, containing a J guide wire, was passed through the incision and the guide wire passed into the tracheal lumen. The cannula was then removed and a 16 French pacemaker lead introducer set, with a peel away Teflon cannula (William Cook Europe Ltd), was advanced over the guide wire into the trachea. The introducer set was removed and a specially designed blind ending polythene catheter (fig 1) was passed into the trachea through the Teflon cannula. The cannula was then peeled away and the catheter was advanced into the affected airway under bronchoscopic vision until the tip lay beyond the lower border of the tumour. A measuring stick with $2 \mathrm{~cm}$ marks (fig 1A) was inserted inside the catheter to facilitate accurate placement. When the correct position had been obtained, the catheter was held in a clamp constructed from delrin (figs $1 B$ and $1 C$ ). The clamp was tied loosely to the neck with gauze ribbon and then secured more firmly with adhesive tape. A dummy radiation source was inserted into the catheter and its position checked by chest radiography (fig 2). Most of the procedures were performed by a physician (PJMG or RMR) after initial instruction in cricothyroid puncture from a surgical colleague (JMH).

AFTERLOADING WITH CAESIUM-137

The patient was transferred to a room adequately shielded for medium dose rate ${ }^{137} \mathrm{Cs}$ sources. The catheter was connected to an afterloading machine (Curietron CGR) into which a ${ }^{137} \mathrm{Cs}$ source train (Amersham International) had been loaded. The length of the source train was such that it extended beyond the proximal and distal margins of the tumour by a distance of $20 \%$ of the total length of the 


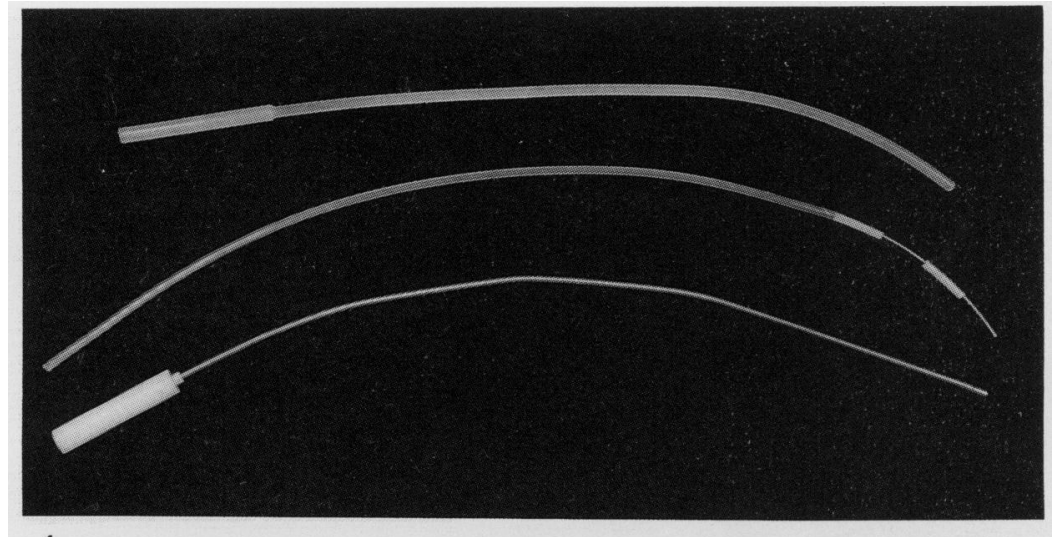

$A$

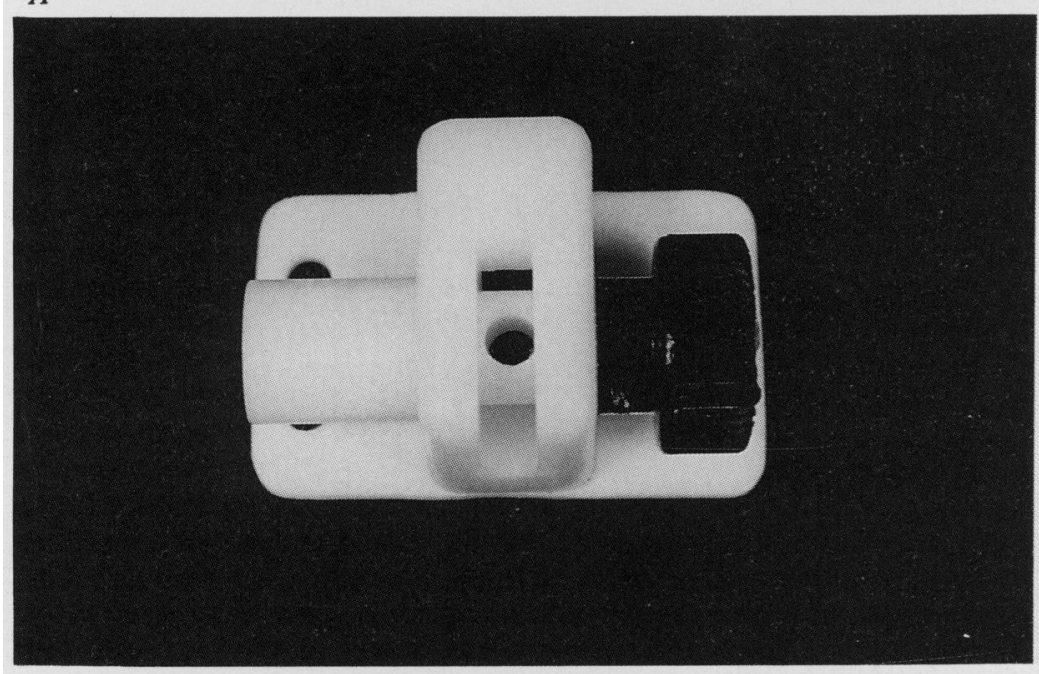

B

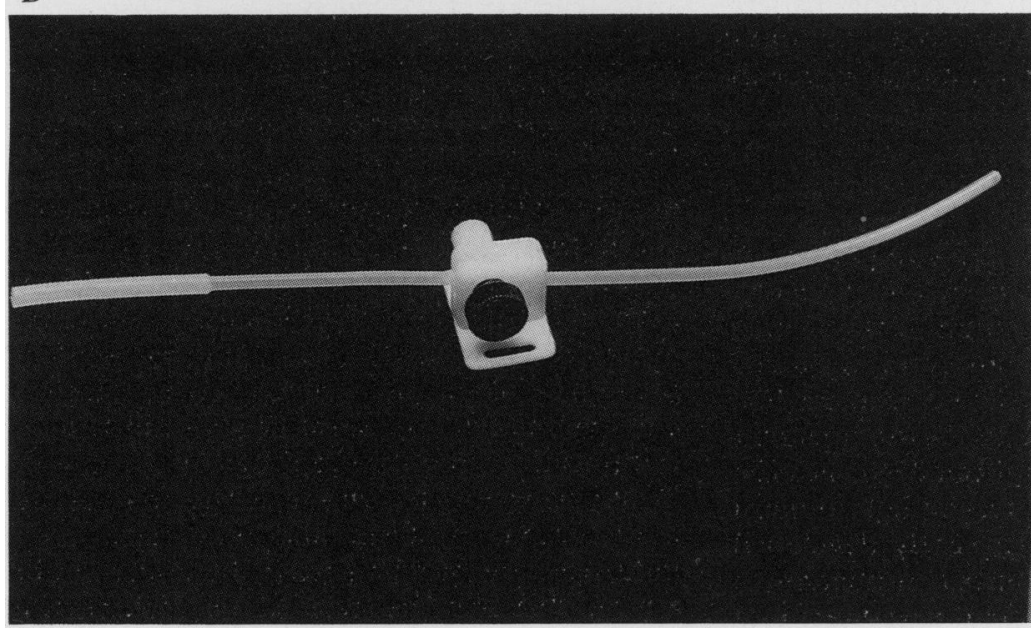

C

Figure 1 The afterloading catheter, measuring stick, dummy source $(A)$ and delrin clamp $(B)$ are shown. The afterloading catheter was constructed from a $308 \mathrm{~mm}$ length of transparent polythene tubing (external diameter $4.4 \mathrm{~mm}$, internal diameter $3.5 \mathrm{~mm}$ ) and was inserted in a peg within the clamp and then held in place by tightening a thumb wheel (C). The angle made by the catheter with the clamp could be varied by rotating the peg along its length, a slot in the body of the clamp accommodating movement of the catheter. The catheter had a $6 \mathrm{~mm}$ extension (internal diameter $4.4 \mathrm{~mm}$, external diameter $5.3 \mathrm{~mm}$ ), which provided a connection for the source transfer tube of the afterloading machine.

\section{Patients}

Ten patients (six male) have so far been treated with endobronchial radiotherapy after initial laser treatment. Their average age was 59 (range 43-79) years. Tissue obtained for histological examination showed squamous cell carcinoma in eight patients and large cell carcinoma in two. The sites of obstruction were the right main bronchus extending into the trachea (two cases), left main bronchus (three cases), right main bronchus (three cases), and right intermediate bronchus (two cases). Endobronchial obstruction was complete and associated with distal collapse in four patients. Seven patients had previously been treated with external radiotherapy either alone (three cases) or in combination with surgery (one case) or chemotherapy (three cases). Two patients had not received any previous treatment and one had previously undergone a lobectomy.

\section{Response to treatment}

Airway calibre was improved by laser treatment in every patient and the lung re-expanded in the four patients with atelectasis. Recurrent collapse, however, developed in one patient within days of endobronchial radiotherapy as a result of obstruction caused by fibrinaceous exudate. This was cleared by a toilet bronchoscopy but the lung did not re-expand.

In the remaining nine patients symptoms of cough and breathlessness were much improved. Two patients with tracheal obstruction had been bed bound before treatment and the remaining seven had been house bound. After treatment all were able to return to active and independent lifestyles until relapse or death. Symptomatic improvement was associated with significant improvements (paired Student's $t$ test) in mean values of peak expiratory flow (PEF) and spirometric values (table). Patients with tracheal obstruction showed the most striking improvements in PEF, whereas those with endobronchial obstruction had more striking improvements in forced vital capacity (FVC).

The nine patients who responded to treatment were followed at regular intervals; their main symptoms were documented and measurements of lung function were repeated (fig 4). Two patients died from massive haemoptyses after two and four weeks with no clinical evidence of airway obstruction. Obstruction due to recurrent intraluminal tumour developed in six patients, however, and required further laser treatments after eight,

Mean (SD) values for peak expiratory flow and spirometric indices before and after laser treatment and endobronchial radiotherapy in nine patients

tumour. Treatment times varied from 5 to $7 \cdot 5$ hours for delivering doses ranging from 2000 to $3000 \mathrm{cGy}$ at a distance of $0.5 \mathrm{~cm}$ from the hottest point of the source (fig 3). At the end of treatment the source was retrieved and the afterloading catheter removed. The minitracheotomy was covered with gauze and allowed to heal.

\begin{tabular}{|c|c|c|c|}
\hline & Before & After & $p^{\star}$ \\
\hline PEF (1/min) & $197 \quad(54)$ & $245 \quad(70)$ & 0.03 \\
\hline $\mathrm{FEV}_{1}(\mathrm{l} / \mathrm{s})$ & $1.46(0.47)$ & $1.71(0.43)$ & $<0.01$ \\
\hline FVC (1) & $2 \cdot 18(0.64)$ & $2.78(0.50)$ & 0.01 \\
\hline
\end{tabular}

*Values compared by paired Student's $t$ test.

PEF-peak expiratory flow; $\mathrm{FEV}_{1}$-forced expiratory volume in one second; FVC-forced vital capacity. 


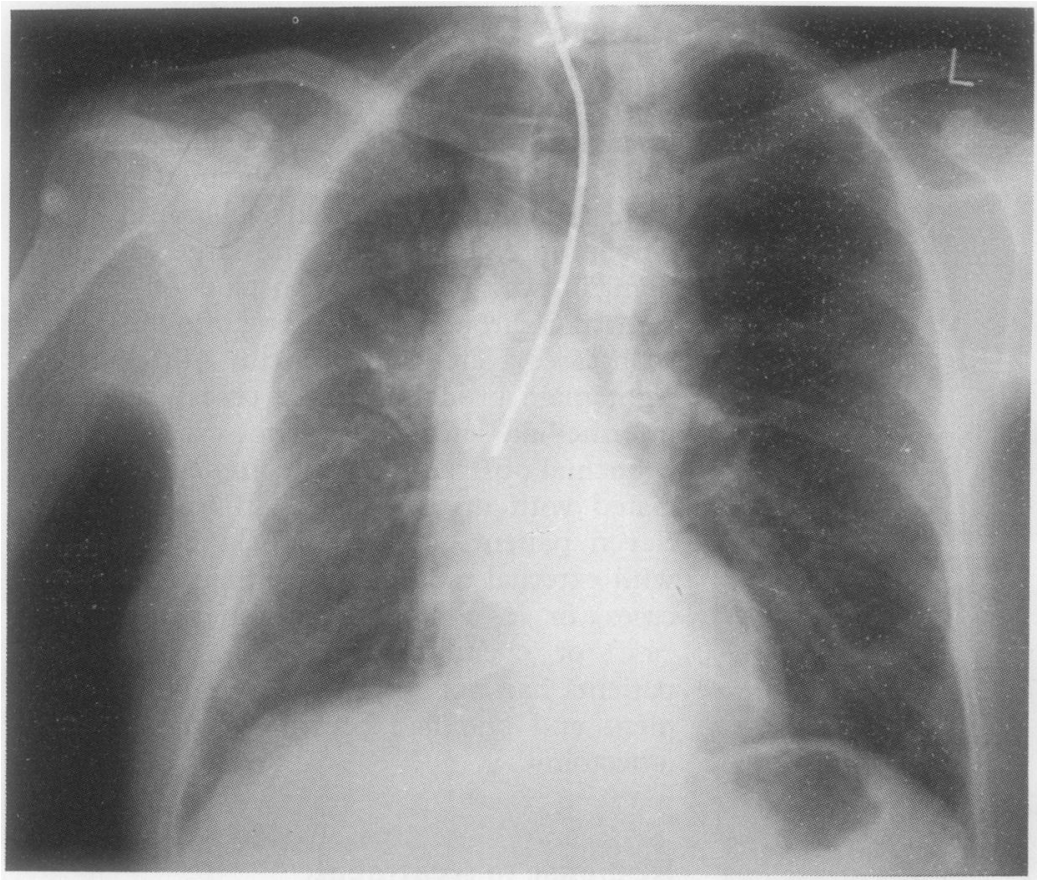

Figure 2 Chest radiograph showing the position of the afterloading catheter and dummy source. The right main bronchus was occluded by squamous cell carcinoma but the lung re-expanded after laser treatment. The tip of the catheter lies below the lower border of the tumour in the right intermediate bronchus. Further details of this patient are given in figure 4 .
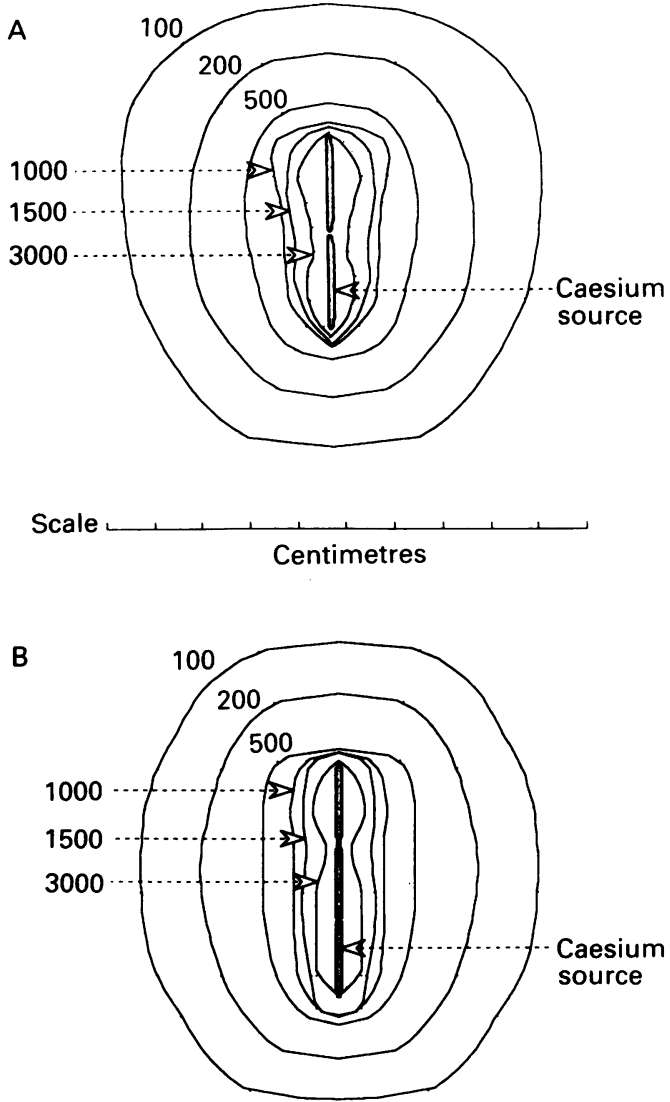

$10,11,26,36$, and 77 weeks. Two of these patients developed recurrent tracheal obstruction after laser therapy and required tracheal stents; details of one of these patients have been reported elsewhere. ${ }^{7}$ The remaining patient developed a stricture of the treated airway after 20 weeks (see below), which was managed with an expandable metal stent. ${ }^{8}$

\section{Complications}

Complications were associated with placement of the afterloading catheter in two patients. One developed surgical emphysema, which resolved rapidly without compromising treatment and did not recur when endobronchial radiotherapy was repeated. In the other patient cricothyrotomy was complicated by arterial bleeding. Although this responded to local pressure, the patient subsequently developed laryngeal oedema and required intubation to protect the airway. The oedema was thought to have been caused by the irritant effects of jet ventilation and instrumentation, which were more protracted than usual as a result of the bleeding. Treatment was performed successfully one week later without recurrence of this problem.

One patient developed a stricture within the treated airway 20 weeks after endobronchial radiotherapy. This was attributed to postirradiation fibrosis as residual tumour was not seen at bronchoscopy and computed tomography provided no evidence of extrinsic compression by extraluminal tumour.

\section{Evaluation of the technique}

Treatment with ${ }^{137} \mathrm{Cs}$ was generally well tolerated. The transtracheal catheters did not
Scale

$$
\text { Centimetres }
$$

Figure 3 Isodose distributions of $4 \mathrm{~cm}(A)$ and $6 \mathrm{~cm}$ (B) caesium-137 sources. The radiation dose falls off steeply with distance from the source, allowing peribronchial tissues but negligible doses to surrounding lung parenchyma. The numbers give the total dose in $c G y$ delivered after 7.5 hours.

interfere with the patient's ability to swallow or speak and did not exacerbate symptoms of cough or breathlessness. Laser treatment may have been of value in this latter respect by providing an optimal airway. Minitracheotomy incisions were associated with surgical emphysema and laryngeal oedema, but these complications resolved rapidly and were seen in only two patients. Laryngeal oedema was the most serious problem and may have been influenced by bleeding from the cricothyroid artery; this may be avoided by making the incision immediately adjacent to the upper border of the cricoid cartilage. The other

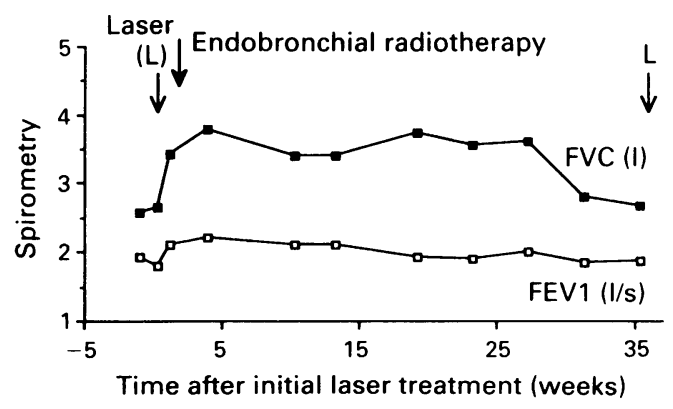

Figure 4 Spirometric changes in a patient whose lung re-expanded after laser treatment and endobronchial radiotherapy (see fig 2). The lung remained aerated for about eight months. Laser treatment was repeated after nine months. therapeutic doses to be delivered to the tumour and 
possible complication of treatment was a stricture which developed within the treated airway 20 weeks after endobronchial radiotherapy. This was attributed to the high local radiation dose and was not thought to represent a specific complication of the technique.

The intention of endobronchial radiotherapy was to prolong the period of symptomatic relief obtained with laser treatment. Patients with non-small cell lung cancer whom we have previously treated with the laser have usually required repeat treatments after intervals of six to eight weeks. ${ }^{6}$ The symptom free intervals appear to be particularly brief in patients with lung collapse and have been reported by other workers to average less than one month. ${ }^{9}$ Without a prospective clinical trial endobronchial radiotherapy cannot, of course, be claimed to have extended this period of palliation. Nevertheless, the symptom free intervals observed in our patients with both partial and complete endobronchial obstruction (see, for example, fig 4) compare favourably with those reported when laser treatment is given alone. ${ }^{69}$

The technique that we have developed for delivering endobronchial radiotherapy is more invasive and time consuming than methods using high activity sources. It also requires a general anaesthetic and a brief stay in hospital. We have since been able to simplify the technique, however, by inserting the afterloading catheters at the end of the session in which laser treatment is administered. The period in hospital is therefore similar to that of patients receiving laser therapy alone.

The advantages of our technique arise from its relatively low capital cost and ready availability. Although an afterloading device was used in the present study, the sources could have been loaded manually. This would reduce the equipment costs further, though it would necessitate some radiation exposure for the staff. As facilities for treating gynaecological malignancy with ${ }^{137} \mathrm{Cs}$ are widely available, this method of treatment could be set up at little extra expense in many hospitals in Britain.

We would like to thank Mr S B Field for designing and constructing the clamps and catheters which were used for ${ }^{137} \mathrm{Cs}$ afterloading. We are also indebted to Sister Tsang for her assistance with laser therapy and the insertion of afterloading catheters.

1 Ledingham SJM, Goldstraw P. Diathermy resection and radioactive gold grains for palliation of obstruction due to recurrence of bronchial carcinoma after external irradiation. Thorax 1989;44:48-51.

2 Schray MF, McDougall JC, Martinez A, Brutinel WM. Management of malignant airway compromise with laser and low dose rate brachytherapy: the Mayo Clinic and low dose rate brachytherapy

3 Macha HN, Koch K, Stadler M, et al. New technique for treating occlusive and stenosing tumours of the trachea and main bronchi: Endobronchial irradiation by high dose Iridium-192 combined with laser canalisation. Thorax 1987;42:511-5.

4 Mehta M, Shahabi S, Jarjour N, Steinmetz M, Kubsad S. Effect of endobronchial radiation therapy on malignant endobronchial obstruction. Chest 1990;97:662-5.

5 Burt PA, O'Driscoll BR, Notley HM, Barber PV, Stout R. Intraluminal irradiation for the palliation of lung cancer with the high dose rate micro-Selectron. Thorax 1990; 45:765-8.

6 George PJM, Pattison J, Al Jarad N, Tsang O, Whitburn RH, Goldhill DR, et al. Preliminary experience with the $1.32 \mu \mathrm{m}$ neodymium YAG laser in the treatment of tracheobronchial malignancy. Lasers in Medical Science 1991;6:407-13.

7 George PJM, Irving JD, Mantell BS, Rudd RM. Covered expandable metal stent for recurrent tracheal obstruction. Lancet 1990;335:582-4.

8 Simonds AK, Irving JD, Clarke SW, Dick R. Use of expandable metallic stents in the treatment of bronchial obstruction. Thorax 1989;44:680-1.

9 Gelb AF, Epstein JD. Laser in treatment of lung cancer. Chest 1984;86:662-6. 